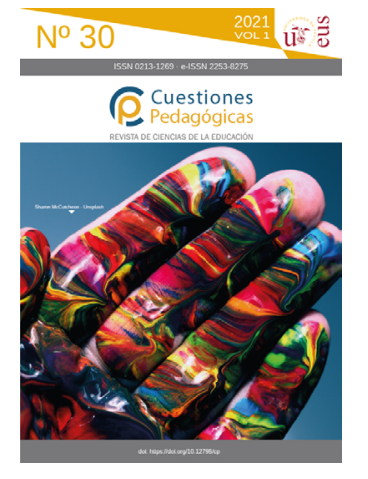

\title{
Libros de texto y enculturación del español en Costa de Marfil
}

\author{
Textbooks and enculturation of spanish foreign language \\ in Ivory Coast
}

Recibido: 15/02/2021 | Revisado: 13/05/2021 | Aceptado: 08/06/2021 |

Online first: 10/06/2021 | Publicado: 01/07/2021

\begin{abstract}
Resumen: La interculturalidad es un concepto al que nos hemos acostumbrado mucho en las últimas décadas, en un mundo globalizado orientado a fomentar el encuentro y contacto de culturas en todos sus rincones. En el ámbito educativo, el enfoque intercultural alienta el respeto a la cultura del otro y, en lo que atañe a la enseñanza-aprendizaje de lenguas extranjeras en particular, supone tener en cuenta las realidades socioculturales del alumnado para hacer más significativo el aprendizaje. En Costa de Marfil, hasta finales de los años 90 del siglo pasado, los libros de texto de Español Lengua Extranjera (en adelante ELE) elaborados y editados en Francia para sus antiguas colonias plantearon el aprendizaje del español como un simple proceso de aculturación del alumno marfileño. En 1998, la colección de manuales Horizontes elaborados por profesionales africanos en la transición hacia el enfoque comunicativo inaugura una era de enculturación del español en una dinámica francamente intercultural. Hoy, iYa estamos! (2018, 2019) y iMás allá! (2020), los nuevos libros de texto de ELE de enseñanza media y secundaria confirman esta dinámica. Al aprender una lengua extranjera y su cultura (aculturación) en contexto de no inmersión, el alumno ha de aprender a hablar también de sus propias realidades en esta lengua (enculturación), lo cual implica la incorporación de nuevos conceptos en el léxico de la lengua meta.
\end{abstract}

Palabras clave: Aculturación, enculturación, interculturalidad, interlengua, libro de texto, aprendizaje de lengua extranjera.
Abstract: Interculturality is a concept to which we have become very accustomed in recent decades, in a globalized world aimed at promoting the encounter and contact of cultures in all its corners. In the educational field, the intercultural approach encourages respect for the culture of the other and, as regards the teaching-learning of foreign languages in particular, means taking into account the socio-cultural realities of the students to make learning more meaningful. In Ivory Coast, until the end of the 90s of the last century, the Spanish Foreign Language (onwards SFL) textbooks elaborated and published in France for its former colonies proposed the learning of Spanish as a simple process of acculturation of the Ivorian student. In 1998, the collection of manuals Horizontes produced by African professionals in the transition towards a communicative approach inaugurated an era of enculturation of Spanish in a frankly intercultural dynamic. Today, iYa estamos! $(2018,2019)$ and iMás allá! (2020), the new SFL textbooks for middle and high school confirm this dynamic. When learning a foreign language and its culture (acculturation) in the context of non-immersion, the student has to learn to talk also about his own realities in this language (enculturation), which implies the incorporation of new concepts in the lexicon of the target language.

Keywords: Acculturation, enculturation, interculturality, interlanguage, textbook, foreign language learning. 
Partiendo de la evidencia de que pese a "la gran variedad de recursos educativos existentes en el mercado y los avances producidos en el campo tecnológico, la práctica de la enseñanza se sigue apoyando mayoritariamente en el libro de texto" (García y Caballero, 2005, citados por Fernández Palop y Caballero García, 2017, p. 202), entendemos que el análisis del libro de texto en la investigación científica no está pasado de moda. Más bien tiene toda su relevancia en un contexto socioeducativo como el de Costa de Marfil donde los avances tecnológicos no resultan todavía tan importantes como para permitir que se pueda prescindir del material impreso, si es que tales avances hayan logrado desterrar el libro de texto en los contextos más desarrollados.

Lo confirma la edición reciente de dos manuales de español para el primer ciclo (ESO) y el segundo ciclo (Bachillerato) de enseñanza secundaria marfileña por parte de la Sección Español del Departamento de la Pedagogía y la Formación Continua (DPFC) adscrito al Ministerio de Educación nacional. Se trata de $i$ Ya estamos! $4^{\circ}$ (Niango et al., 2018), iYa estamos! $3^{\circ}$ (Niango et al., 2019) para los niveles iniciales (Tercero y Cuarto), y de las tres versiones de ¡Más allá! para Primero (Yao et al., 2020), Segundo (Meless et al., 2020) y Último (Yao et al., 2020) de Bachillerato. O sea, cinco libros de texto elaborados únicamente para la enseñanza-aprendizaje del español en Costa de Marfil, y que constituyen el corpus del presente estudio.

Al emprender esta investigación tras otras colectivas sobre la temática del libro de texto (Djandué, Djè y Kumon, 2019; Bekale et al., 2020; Djè, Kumon y Djandué, 2020), pretendemos responder a los siguientes interrogantes: ¿cómo después del largo periodo de los libros de texto editados en Francia, y que no dedicaban ninguna línea a las realidades africanas, ¡Ya estamos! y ¡Más allá! fortalecen la enculturación de los manuales de ELE inaugurada por Horizontes $(1998,1999,2000,2001,2002)$ ? ¿Y cómo, a su vez, la enculturación de los manuales desemboca en la de la misma lengua española al usar esta para expresar realidades propias de sus aprendientes marfileños en un contexto geográfico y sociocultural muy distinto?

Para aportar respuestas a estos interrogantes, organizamos nuestra reflexión en tres apartados. El primer apartado se dedica a la fundamentación teórica del estudio en torno a tres conceptos claves: la interculturalidad, la aculturación y la enculturación como diferentes modos de enfocar la enseñanza-aprendizaje de una LE de cara a su cultura y a la cultura de sus aprendices no nativos. El segundo apartado aporta más informaciones sobre el corpus del estudio antes de describir el método de investigación adoptado para alcanzar los objetivos marcados. Por fin, en el tercer y último apartado exponemos los resultados y los discutimos.

\section{Fundamentación teórica}

Tal como formulado, el tema del estudio pone la cultura en el centro del mismo, entendiendo el hecho cultural como "el resultado de un conjunto de prácticas sociales y de un conjunto de discursos construidos sobre dichas prácticas [...], una realidad [...] múltiple, plural y concreta que depende de factores relativos al individuo y al entorno al que este pertenece" (Solís, 2012, p. 2). Es un todo en el que la persona está inmersa; incluye aspectos tan característicos como la forma en que viste, en que habla, en que se comporta, sus ideas, sus conocimientos, etc. (Medios y Cultura, 2006, citados por Solís, 2012, p. 2). El enfoque intercultural se nutre de estos aspectos muy humanos de la cultura.

\section{El enfoque intercultural en el aprendizaje de ELE}

Siguiendo a Hernández y Valdez (2010, pp. 92-94), entre otros autores de referencia, la enseñanza de la cultura en las clases de LE se ha venido planteando según diferentes perspectivas a lo largo del tiempo, siendo estas las cuatro principales:

a) El enfoque de transmisión de información: proporciona al estudiante información cultural: estadísticas, instituciones, información de corte académico o intelectual sobre literatura, bellas artes, cultura popular enfocada a costumbres, hábitos y folklore de la vida diaria. Ha sido criticado por 
ofrecer solamente datos, más que entendimiento de las actitudes y valores de la cultura meta.

b) El enfoque contrastivo de culturas: pretende que los estudiantes conozcan y encuentren semejanzas y diferencias entre su propia cultura y la cultura de la lengua meta. Pero simplifica la riqueza y la variedad de las culturas a unos cuantos aspectos.

c) El enfoque basado en la competencia comunicativa: interviene en la década de los 80 del siglo pasado, con el surgimiento y aceptación del enfoque comunicativo en la enseñanza de LE y como consecuencia de la importancia concedida a la cultura en el currículum. Así, se crea la conciencia de integrar en los programas algo más que conocimiento lingüístico para dominar una LE en una situación real.

d) El enfoque intercultural: es el que actualmente se promueve en la enseñanza de LE. Su objetivo fundamental es desarrollar la competencia intercultural en los alumnos, en un proceso que enfatiza las mezclas, la comunicación, los conflictos y los préstamos entre las diversas culturas.

El enfoque intercultural le debe mucho al enfoque comunicativo. Los métodos anteriores no consideraban la cultura como consustancial a la lengua. Los contenidos lingüísticos tenían una presencia predominante y un mayor protagonismo en los programas, mientras que los contenidos culturales aparecían solo como valores agregados y de menor interés. El enfoque comunicativo trae consigo la superación de la competencia lingüística por la competencia comunicativa que la incluye y también abarca las competencias discursiva, estratégica y sociocultural según las propuestas de Canale y Swain (1980) (Solís, 2012, p. 3).

Sin embargo, expresa García Benito (2009, pp. 499-500), el enfoque intercultural supone ir más allá de los contenidos gramaticales, funcionales y culturales. Al tiempo que busca desarrollar tales conocimientos, fomenta una serie de valores y actitudes con el objetivo de formar a hablantes e intermediarios interculturales. Los materiales diseñados al respecto, en vez de aquellos que se centran en proporcionar información sobre la cultura objeto de estudio, brindan a los alumnos la oportunidad de reflexionar sobre sus propias culturas. De ahí que asistimos a una nueva superación, la de la competencia comunicativa por la competencia intercultural como una competencia más amplia y compleja todavía (Barros García y Kharnásova, 2012).

Sintetizando de diferentes autores, Hernández y Valdez (2010, pp. 92-94) afirman que la competencia intercultural consiste en conocer y comprender otros sistemas culturales, sin abandonar los propios. En las aulas de ELE observamos encuentros de diversas culturas; los alumnos llegan con conocimientos, conductas y actitudes cimentados en su propia cultura y esta representa su punto de referencia en el aprendizaje del nuevo idioma. Pero, porque "el comportamiento en una cultura no debe ser juzgado con los patrones de otra", hay que reconocer con Níkleva (2009, p. 40) que el alumno de una LE debe estudiar también su cultura en nombre de lo que ella denomina "la convivencia intercultural".

De todo lo que antecede, se desprende que la enseñanza de la cultura en las aulas de LE ha privilegiado primero la sola cultura de la lengua meta (enfoque de transmisión de información) antes de empezar a contemplar también la cultura de los alumnos que aprenden dicha lengua (enfoque contrastivo de culturas, enfoque basado en la competencia comunicativa y enfoque intercultural). Con el actual enfoque intercultural en un mundo cada vez más globalizado, esta evolución supone que el aprendizaje de ELE ya no se puede plantear únicamente como un proceso de aculturación; debe ser también necesariamente un proceso de enculturación de la lengua meta, en especial cuando el estudio se desarrolla en otro contexto geográfico y sociocultural.

\section{Aculturación y enculturación en clase de ELE}

La aculturación es el proceso por el cual el contacto entre grupos culturales diferentes lleva a la adquisición de nuevos patrones culturales por parte de uno, o los dos grupos, con la adopción de parte o toda la cultura del otro grupo (Pérez Francés, 2002, p. 395). En cuanto a la enculturación (o inculturación), es el hecho de "encarnarse en un pueblo de cultura distinta", lo cual supone "indigenizarse con el indígena, indianizarse con el indio, africanizarse con el africano, españolizarse con el español, europeizarse 
con el europeo, pero bautizando valores nativos con nuevos valores que se aportan" (Rodríguez Díez, 2004, pp. 28-29).

La adquisición de una LE se da como una forma de aculturación o adaptación a otra cultura (Moreno Fernández, 2004, p. 296), pero también conlleva el movimiento complementario de adaptarse la lengua estudiada a las realidades socioculturales de los aprendices. "La noción de interculturalidad introduce una perspectiva más dinámica de las culturas" (Pérez Francés, 2002, p. 394) que conviene promover explícitamente en las aulas de LE. Siendo el aula de LE un espacio de contacto de lenguas-culturas diferentes, al aprender la nueva lengua el alumno automáticamente se impregna de su cultura (aculturación inconsciente del individuo) y al mismo tiempo busca adaptarla a su propia cultura (enculturación inconsciente del idioma).

Se perfila en ello una superación del concepto de interlengua para postular el de intercultura. El aprendiz de una LE no solo se mueve entre dos o más lenguas sino también y sobre todo entre dos o más culturas, en nombre de la arriba referida consustancialidad entre lengua y cultura. Sus producciones orales o escritas no solo se caracterizan por llevar la influencia de su lengua materna sino, más allá, de su cultura nativa. En realidad, la influencia de la(s) primera(s) lengua(s) en la interlengua no se limita a lo lingüístico. Además, no todo en la interlengua se puede considerar errores intralingüísticos o interlingüísticos. En base a esta evidencia, postulamos la interlengua particular de los usuarios marfileños de ELE como el punto de partida de un español marfileño asumible (Djandué, 2018; Bayoko, 2021) que, de alguna forma, se refleja en los nuevos libros de texto de ELE.

Para comprender la transcendencia de esta evolución, téngase en cuenta que en Costa de Marfil, hasta finales de los años 1990, los libros de texto elaborados en Francia llegaban a nuestras aulas con un planteamiento del aprendizaje de ELE radicalmente como una forma de aculturación al no contemplar las realidades africanas. Los manuales Horizontes $(1998,1999,2000,2001,2002)$ cambian el rumbo en la era del enfoque comunicativo y visibilizan por primera vez a África a través de sus ciudades (Kampala o Dar-Es-Salam...), su onomástica (Ayélé, Dovi, Traoré, Abou, Fatou...), sus mujeres, figuras emblemáticas como la de Nelson Mandela, sus realidades históricas y políticas (esclavitud, colonización, guerras, Unión Africana...), etc. (Djè, 2014, pp. 187-204). El alumnado marfileño, en concreto, se identifica con elementos culturales como el matrimonio tradicional, el poro de los Senufó, la fiesta del ñame de los pueblos Akán, la toponimia local representada por Plateau, el barrio administrativo de Abidjan, o el barrio residencial de Treichville.

En la opinión de Djè, Kumon y Djandué (2020, p. 358), el alumno marfileño toma entonces consciencia no solo de la realidad cultural propia sino también de la ajena ya desde los libros de texto en una situación de no inmersión. Estos investigadores ven necesario que el alumnado pueda expresar todo lo que le rodea, sus condiciones de estudio, sus creencias y valores en español sin pasar por alto la cultura hispánica. Estamos hablando de enfoque comunicativo y, según Djandué, Djè y Kumon (2019, p. 101), así es como se le ofrece "al alumnado posibilidades de conversación en ELE", ya que las personas usan las lenguas para hablar de lo que ven y viven a diario. Por su parte, Bekale et al. (2020, pp. 4-5) entienden que la eficaz aplicación del enfoque comunicativo a contextos africanos y su éxito dependen no de la transposición sino de la adaptación de los materiales utilizados a las culturas y entornos educativos meta.

\section{Corpus y método de investigación}

Consolidando la dinámica de adaptación metodológica y cultural iniciada por Horizontes, los nuevos libros de texto de ELE de enseñanza media y secundaria marfileña ( Ya estamos! y iMás allá!) asumen la enculturación del español que, en su caso, tendría como nombre propio marfileñización dentro de un marco más amplio de africanización de los materiales didácticos de ELE. Como lo hemos avanzado, en este apartado presentamos los manuales analizados y describimos el método de investigación. 


\section{Corpus del estudio}

En Costa de Marfil, el estudio del ELE empieza oficialmente en Tercero de ESO (Quatrième en el sistema francés y francófono). El alumno ya aprende inglés desde el primer año y, en Tercero, tiene que elegir el español o el alemán como segunda lengua viva obligatoria. Por lo tanto, son dos niveles de aprendizaje de ELE en ESO y tres en Bachillerato, especialmente para el alumnado de rama literaria. $\mathrm{O}$ sea un total de cinco niveles de aprendizaje del español en la educación media y secundaria marfileña.

Después de dos decenios de uso continuo de los manuales Horizontes en nuestras aulas, el proceso de renovación del libro de texto se inicia en 2018 con iYa estamos! $4^{\circ}$ (Tercero de ESO). El año siguiente se edita iYa estamos! $3^{\circ}$ (Cuarto de ESO).

\section{Tabla 1}

Descripción de los manuales iYa estamos!

\begin{tabular}{|c|c|c|}
\hline Título & ¡Ya estamos! $4^{\circ}$ & ¡Ya estamos! $3^{\circ}$ \\
\hline Disciplina & Español Lengua Extranjera & Español Lengua Extranjera \\
\hline Nivel de estudio & Tercero de ESO & Cuarto de ESO \\
\hline Edad de los alumnos & 12-15 años & 14-17 años \\
\hline Editorial & NEI/CEDA & NEI/CEDA \\
\hline Año de publicación & 2018 & 2019 \\
\hline Autores & $\begin{array}{l}\text { Niango Aloboué Camille, Melles } \\
\text { Isidore Aimé, Yepri ADjè Jean-Bap- } \\
\text { tiste, Allou Oi Allou, Silué Souleymane }\end{array}$ & $\begin{array}{l}\text { Niango Aloboué Camille, N'guessan } \\
\text { N'dri Henriette, Kouassi Kouamé, } \\
\text { N'goran Yao Victor, Yapi François } \\
\text { d'Assise }\end{array}$ \\
\hline Número de páginas & 87 & 111 \\
\hline Encuadernación & $\begin{array}{l}\text { Tapa blanda, multicolor con domi- } \\
\text { nante azul }\end{array}$ & $\begin{array}{l}\text { Tapa blanda, multicolor con domi- } \\
\text { nante morado }\end{array}$ \\
\hline
\end{tabular}

Fuente. Djè, Kumon y Djandué (2020, p. 353).

En 2020 se culmina el proceso de renovación del libro de texto de ELE aportando dos grandes innovaciones en comparación con lo que ocurrió con Horizontes. En primer lugar, se abandona el título ¡Ya estamos! de los niveles iniciales y se acuña uno nuevo para el Bachillerato (¡Más allá!), el cual significa claramente un paso adelante en el aprendizaje del idioma. En segundo lugar, las tres versiones de ¡Más allá! se editan en el mismo año, acortando así la duración del proceso de renovación.

\section{Tabla 2}

Descripción de los manuales iMás allá!

\begin{tabular}{|c|c|c|c|}
\hline Título & ¡Más allá! 2de & ¡Más allá! 1ère & ¡Más allá! Tle \\
\hline Disciplina & ELE & ELE & ELE \\
\hline Nivel de estudio & 10 de bachillerato & 2o de bachillerato & Último de bachillerato \\
\hline Edad de los alumnos & 15-16 años & 16-17 años & 17-20 años \\
\hline Editorial & NEI/CEDA & NEI/CEDA & NEI/CEDA \\
\hline Año de publicación & 2020 & 2020 & 2020 \\
\hline Autores & $\begin{array}{l}\text { Yao Amenan Thérèse Epse } \\
\text { Doumouya, Kouassi Koua- } \\
\text { mé, Allou oi Allou, Kouakou } \\
\text { Née Ikossié Véronique, Tuo } \\
\text { Gofégué }\end{array}$ & $\begin{array}{l}\text { Meless Isidore Aimé, Kouas- } \\
\text { si Kouamé, N'douba Kas- } \\
\text { si, Koffi N'guessan Jules, } \\
\text { Kouadio Yao Jacques }\end{array}$ & $\begin{array}{l}\text { Yao Amenan Thérèse Epse } \\
\text { Doumouya, Kouassi Koua- } \\
\text { mé, Allou Oi Allou, N'goran } \\
\text { Yao Victor, Miézou Tanoh } \\
\text { Hubert Basile }\end{array}$ \\
\hline Número de páginas & 118 & 139 & 138 \\
\hline Encuadernación & $\begin{array}{l}\text { Tapa blanda, multicolor con } \\
\text { dominante rojo }\end{array}$ & $\begin{array}{l}\text { Tapa blanda, multicolor con } \\
\text { dominante verde }\end{array}$ & $\begin{array}{l}\text { Tapa blanda, multicolor con } \\
\text { dominante verdel }\end{array}$ \\
\hline
\end{tabular}

Fuente. Elaboración propia. 
Se nota que el número de páginas de los libros va aumentando a medida que subimos de nivel (87 p., 111 p., 118 p., 139 p. y 138 p.), lo cual supone un lógico incremento progresivo de los contenidos. Todos los autores son marfileños; también son inspectores pedagógicos, excepto Kouakou Née Ikossié Véronique, doctora y profesora de literatura y civilización española en la Escuela Normal Superior de Abidjan, jefa de la Sección Español del Departamento de Lenguas, coautora de ¡Más allá! 2de en el marco de una colaboración entre los profesionales encargados de la formación inicial del profesorado marfileño de ELE de enseñanza media y secundaria y los encargados de su formación continua (Djandué, 2019) ${ }^{1}$.

\section{Método de Investigación}

Completada la actualización del material didáctico iniciada en 2018, los investigadores estamos en condiciones para hacer análisis más pertinentes del conjunto de los cinco libros de texto según las perspectivas que nos parezcan oportunas. Hablando del aporte de los libros de texto en la enculturación del español en Costa de Marfil, nos referimos a cómo el corpus descrito, al dar cabida a la cultura marfileña, refleja cierta adaptación de la lengua española a las características sociolingüísticas de sus aprendices marfileños, a cerca de 6000 kilómetros de España y dentro de un ambiente libresco de gran convivencia intercultural. Privilegiando el método cualitativo de recogida y análisis de datos, hemos recorrido cada uno de los cinco componentes del corpus para buscar en todas sus páginas elementos de cultura marfileña. Los resultados de esta operación se exponen y discuten en el último apartado del trabajo.

\section{Resultados y discusión}

\section{Resultados de la investigación}

Los resultados de esta investigación se presentan en dos tablas. En cada tabla se recogen unos elementos culturales y sus ilustraciones, marcando entre paréntesis las páginas donde estas se hallan en los manuales. La primera tabla combina los resultados de los dos libros de texto de primer ciclo (ESO) y la segunda tabla los resultados de los tres manuales de segundo ciclo (Bachillerato).

Se observa una fuerte presencia de la onomástica marfileña. Los nombres y apellidos nativos remiten a todas las regiones de Costa de Marfil y sus pueblos autóctonos: el centro (Akissi, Konan, Kouassi, etc.), el este (Tano, Amonkuá, etc.), el oeste (Tapé, Gondo, Zézé, etc.), el sur (Mobio, Lohouess, Badjo, etc.) y el norte (Kolo, Sita, Awa, etc.); pero también a la importante población de origen africana presente en el territorio nacional: Sako Abdul (de Mali). Esta diversidad onomástica casa no solo con la que prevalece a nivel sociocultural en el país, sino también con los ambientes sociales variopintos de nuestras aulas de ELE.

\section{Tabla 3}

Elementos culturales marfileños en ;Ya estamos!

\begin{tabular}{|c|c|c|}
\hline Elementos culturales & En iYa estamos! $4^{\circ}$ & En iYa estamos!3o \\
\hline Onomástica & $\begin{array}{l}\text { Akissi (p.18); Dido, Sié, Zran, Danho } \\
\text { (p.20); Adjoba (p.22); Konan (p.24); } \\
\text { Gondo, Tanoh (p.26); Zézé (p.27); } \\
\text { Kolo, Aka (p.30); Loua (p.38); Badjo, } \\
\text { Lohouess (p.42); Ayaba (p.48); } \\
\text { Mobio, Sita, Awa (p.58); Yao (p.60); } \\
\text { Kouassi (p.77); etc. }\end{array}$ & $\begin{array}{l}\text { Kouam, Sako Abdul, (p.16-17); Samy } \\
\text { (p.17); Ema (p.20); Sita (p.37); Tano, } \\
\text { Amonkuá (p.49); Tapé, Zokú (p.50); } \\
\text { Zenabou, N'zue (p.72); Koné, Kouak- } \\
\text { ou (p.86); etc. }\end{array}$ \\
\hline Toponimia & $\begin{array}{l}\text { Arrah, Ferkessédougou (p.26); Kor- } \\
\text { hogo (p.27); Abidjan, Adjamé, Abobo, } \\
\text { Zoo, Hospital Militar de Abidjan (p.30); } \\
\text { Plateau, Cocody (p.54); etc. }\end{array}$ & $\begin{array}{l}\text { Korhogo (p.16); Adzopé, Man (p.17); } \\
\text { Abidjan, N'sisso (p.32); Betié, puente } \\
\text { de la Comoé (p.49); etc. }\end{array}$ \\
\hline
\end{tabular}

1 Se trata respectivamente de la Sección Español del Departamento de Lenguas de la Escuela Normal Superior de Abidjan y de la Sección Español del Departamento de la Pedagogía y de la Formación Continua (DPFC) con sus diferentes antenas repartidas en la geografía nacional. 


\begin{tabular}{|l|l|l|}
\hline Valores y actitudes & La figura del abuelo (p.28) & $\begin{array}{l}\text { El saludo tradicional, el saludo entre } \\
\text { adolescentes (saludo juvenil) y el } \\
\text { saludo formal (p.25); la escolarización } \\
\text { de las niñas, la figura de la abuela } \\
\text { (p.32); pedir perdón (p.73, 77); etc. }\end{array}$ \\
\hline Figuras & $\begin{array}{l}\text { Marie Josée Talou, Muriel Ahouré } \\
\text { (p.77) }\end{array}$ & Drogba (p.63) \\
\hline Fiestas y tradiciones & Fiesta del ñame (p.26) & $\begin{array}{l}\text { El Zaouli de los gouro (p.51); Día de } \\
\text { mercado (p.65); Paquinou (p.67) }\end{array}$ \\
\hline Cultivos y comidas & Attiéké (p.54) & $\begin{array}{l}\text { El Zaouli de los gouro (p.51); Día de } \\
\text { mercado (p.65); Paquinou (p.67) }\end{array}$ \\
\hline
\end{tabular}

Fuente. Elaboración propia.

Los elementos culturales en su conjunto contribuyen a educar en valores y, lo más importante todavía, permiten crear en los niveles iniciales de aprendizaje una situación de familiaridad entre el alumnado y la lengua meta al hacer que esta parezca menos extranjera de lo que es en realidad. Tal es, en efecto, el primer gran beneficio pedagógico de usar el ELE para expresar realidades socioculturales marfileñas en los inicios del aprendizaje. Y, al proceder de este modo, el español enseñado y aprendido en un otro contexto geográfico y cultural se enriquece de conceptos nuevos que implican naturalmente su marfileñización.

Al respecto, no pasa desapercibida la manera de escribir Amonkuá (nativamente Amonkoua) y Zokú (nativamente Zokou) en un marco general de vacilación ortográfica por parte de los autores de los libros de texto, pues estos no aplican sistemáticamente a todos los términos marfileños el mismo esfuerzo de adaptación a la ortografía del español para hacer más rico y original todavía el diálogo intercultural entre la lengua-cultura hispánica y las lenguas-culturas marfileñas. Al fin y al cabo, y parafraseando a Rodríguez Díez (2004, p. 29), enculturarse en este caso significa marfileñizarse con los marfileños, "pero bautizando valores nativos con nuevos valores que se aportan".

Según los autores de los manuales, Akissi Kouamé es la "primera mujer general en el ejército de Costa de Marfil"; Marie Josée Talou y Murielle Ahouré son "dos gloriosas campeonas marfileñas". Por su parte, Euphrasie Yao es "la representante del presidente marfileño en el Consejo de Orientación del Observatorio del Género" en la Unesco; y Adayé Késsiè "es la mayor fiesta del reino Brong" para celebrar "su Año Nuevo".

\section{Tabla 4}

Descripción de los manuales ¡Más allá!

\begin{tabular}{|l|l|l|l|}
\hline Elementos culturales & ¡Más allá! 2de & ¡Más allá! 1ère & ¡Más allá! Tle \\
\hline Figuras & $\begin{array}{l}\text { Akissi Kouamé, Marie } \\
\text { Josée Talou, Murielle } \\
\text { Ahouré (p.72) }\end{array}$ & $\begin{array}{l}\text { Euphrasie Yao (p.48); } \\
\text { Bernard Dadié (1916-2019) } \\
\text { (p.63); Memel-Fotê Harris }\end{array}$ & Alpha Blondy (p.106) \\
\hline Valores y tradiciones & & $\begin{array}{l}\text { Crónica de la Semana } \\
\text { Santa en Costa de Marfil } \\
\text { (p.75); Tejedor de Wara- } \\
\text { niene (p.93) }\end{array}$ & $\begin{array}{l}\text { El matrimonio infantil (p.59); } \\
\text { Bron (Abron), Adayé Késsiè } \\
\text { (p.67); Contra el trabajo } \\
\text { infantil (p.68) }\end{array}$ \\
\hline
\end{tabular}

Fuente. Elaboración propia.

De los manuales de primer ciclo ( Ya estamos!) a los de segundo ciclo ( Más allá!), llama la atención la drástica reducción de las referencias culturales marfileñas explícitas. Y es así porque en las páginas de los tres niveles de iMás allá! se percibe la voluntad de los autores, pasados los años de iniciación a la lengua-cultura meta apoyándose firmemente en las culturas nativas de los aprendientes, de ir más allá de lo marfileño para entrar de lleno en lo hispánico, de ahí sin duda el título de la colección: ¡Más allá!

Pero ello no significa que se haya silenciado la cultura del mundo hispánico en iYa estamos! $4^{\circ}$ y iYa estamos! $3^{\circ}$, todo al contrario. Lo primero que se propone en ambos manuales es un panorama del 
mundo hispanófono: “¡Bienvenidos a España!” (p.6); “¡Hola Hispanoamérica!” (p.10); "Guinea Ecuatorial en pleno desarrollo" (p.12) ( Ya estamos! 4)/ "Las comunidades autónomas" (p.6); "Hispanoamérica" (p.10); "Guinea Ecuatorial" (p.12) ( $i Y a$ estamos! $3^{\circ}$ ). La diferencia fundamental con ¡Más allá! está en que, tras estas informaciones introductorias, se nota la omnipresencia de elementos culturales locales en las páginas sucesivas como para que sirvan de apoyo cognitivo y afectivo al alumnado a la hora de prepararlo a estudiar una nueva lengua y su cultura.

\section{Discusión de los resultados}

Resulta difícil no ver reflejada en cuanto se ha venido comentando la teoría vigotskiana del aprendizaje. Esta habrá influido no solo en la opción metodológica de los expertos marfileños, sino también en la elección y organización de los contenidos en los libros de texto. Lev Vigotsky hace hincapié en la interacción sociocultural como base del desarrollo humano. Hablando de las implicaciones educativas de su teoría, Chaves Salas (2001, pp. 63-64) resalta "la importancia de respetar al ser humano en su diversidad cultural", "partir de los contextos socioculturales de nuestros estudiantes para ofrecerles una educación con sentido y significado".

Esto es lo que procuran hacer los autores de iYa estamos! y ¡Más allá!, quienes presentan sus productos como "una herramienta de enseñanza-aprendizaje-evaluación que toma en cuenta el entorno sociocultural de los aprendientes y los lleva a descubrir las otras áreas geográficas con sus costumbres y tradiciones" ( Ya estamos! y iMás allá!, p. 3, nuestra traducción). Como bien lo notan Bekale et al. (2020, p. 10), " Ya estamos! apunta hacia el fortalecimiento del proceso de enculturación de los contenidos iniciado con Horizontes". Y prosiguiendo,

...si Horizontes fue una aventura africana, iYa estamos! ha empezado como una aventura marfileña. De la antigua colección a la actual, hay una clara evolución desde la africanización de los contenidos hacia su marfileñización en un momento histórico en el cual se promueve cada vez más la integración africana y todos los esfuerzos deberían orientarse a la consecución de este objetivo continental. (Bekale et al., 2020, p. 10)

Este análisis, prospectivo en su época, es anterior a la edición de ¡Más allá!, cuando todos los observadores pensaban que iYa estamos! iba a conservarse como título de los tres manuales de Bachillerato como pasó con Horizontes. Pero al rebautizar la colección de libros de segundo ciclo, la elección del título ¡Más allá! no fue una mera operación cosmética para distinguir los niveles de aprendizaje en la portada de los manuales. Traducía la voluntad de los autores de ir más allá de los elementos culturales marfileños, pues, la "enseñanza del español", "medio de comunicación entre los pueblos", "debería permitir a sus usuarios participar activamente en el desarrollo global” ( $i Y a$ estamos! y ¡Más allá!, p. 3, nuestra traducción).

Habida cuenta del gran protagonismo que tiene el libro de texto en la educación reglada de Costa de Marfil, su enculturación supone una clara opción por el enfoque intercultural en la enseñanza-aprendizaje de ELE. Para Álvarez González (2010, p. 2), "un encuentro intercultural implica no solamente la convivencia de culturas diferentes, la interacción, sino también el reconocimiento y el respeto de la diversidad del "otro" y la profundización de su "yo", es decir, "yo" con y desde el otro". Se trata entonces de contemplar "la diversidad cultural de los alumnos" y adaptar "los materiales y los cursos a las características lingüísticas y culturales de cada lugar" (Moreno Fernández, 2004, citado por Solís, 2012, p. 2).

La incorporación de elementos culturales marfileños en los libros de texto de ELE tiene una implicación lingüística enorme, porque el encuentro entre culturas también es un encuentro entre lenguas. Encuentro entre la lengua española y las lenguas indígenas marfileñas, contacto insoslayable entre el español estándar o formal y ciertas variaciones locales de la lengua meta que, partiendo de la interlengua particular de los ELE-hablantes marfileños muy marcada por la interferencia del francés, su idioma oficial, constituyen para Djandué (2018) y Bayoko (2021) las premisas de un español marfileño perfectamente asumible.

Que el encuentro entre culturas también sea un encuentro entre lenguas es lo que explica, por un 
lado, la vacilación ortográfica de los autores de iYa estamos! y iMás allá! a la hora de escribir nombres o vocablos típicamente marfileños, no sabiendo si adaptarlos todos al sistema acentual español. De haberlo hecho de una forma sistemática, ello hubiera creado una mayor convivencia intercultural en los manuales. Por otro lado, con respecto a variaciones locales del español inducidas por la interferencia del francés, se le debe frases como estas:

- Samy: ¡Felicitaciones, Abdul! [...] (¡Ya estamos! 3, 2019, p. 23)

- Acabáis de estudiar en clase de español, la lección sobre la expresión de la certidumbre con el texto La equidad de género. Durante el estudio del texto, no estaba tu vecino. Te pide aclaraciones. [...] 2. Informa a tu vecino (¡Más allá! 1ère, 2020, p. 47).

Consideramos "felicitaciones" y "vecino" como unos marfileñismos (o galicismos) que, al colarse en nuestros libros de texto, también contribuyen a la enculturación (marfileñización) de los mismos, pero ante todo desde una perspectiva sociolingüística. Para el primer término, por ejemplo, se aclara en "Castellano Actual" (2015) que "en España [...] felicitaciones se usa muy poco y se prefiere enhorabuena". En cuanto al vocablo "vecino", es una traducción literal del francés "voisin" por parte de muchos profesores y alumnos marfileños de ELE, ahí donde, tomando otra vez a España como referencia, conviene decir "compañero (de clase)".

Clausurando esta discusión, nos ha parecido muy significativo un detalle en la página 17 de iYa estamos! $4^{\circ}$. En la rúbrica "LO SABÍAS" donde se presentan los elementos de cultura, hablando de "Nacionalidades", lo primero que apuntan los autores es: "Costa de Marfil: Marfileño/a". He aquí resumida toda la teoría de aprendizaje en la que se basan los libros de texto analizados y, más allá, toda la política de enseñanza-aprendizaje de ELE en Costa de Marfil desde los años 2000. Partir de lo que uno es, ve y vive para acercarse a la nueva lengua y su cultura, siendo esto el primer requisito para que el aprendizaje y conocimiento del ELE produzcan ciudadanos tolerantes y abiertos al mundo global, mediadores interculturales al servicio del diálogo entre los pueblos.

\section{Conclusión}

Horizontes fue "el único manual de español redactado por africanos para africanos", decía uno de los coautores de la colección (Kanga, citado por Djè, 2014, p. 187); iYa estamos! y ¡Más allá! son los primeros manuales elaborados por marfileños para marfileños. Pero al fortalecer la dinámica de enculturación del libro de texto de ELE y, de rebote, la de la misma lengua española, se puede observar que iYa estamos! $\left(4^{\circ}\right.$ y $\left.3^{\circ}\right)$ marfileñiza los contenidos más que Horizontes ( $4^{\circ}$ y $\left.3^{\circ}\right)$; iMás allá! (2de, 1ère y Tle) los africaniza tanto como Horizontes (2de, 1ère y Tle).

La enculturación del ELE se realiza mediante elementos culturales y recursos lingüísticos. Se trata de usar la lengua española para hablar de temas específicos al contexto sociocultural de los aprendices. Si el español es una lengua viva y la aprendemos para comunicar, lo primero es comentar en español lo que somos y vivimos a diario. Se percibe también la preocupación de los autores por hacer que nuestras aulas de ELE no estén desconectadas de las grandes temáticas nacionales, como un mundo ajeno al de los profesores y alumnos. Así es como el tema de la reconciliación nacional, por poner un caso, se perfila en la gran diversidad de la onomástica y la toponimia locales. El mensaje subyacente es que formamos un solo pueblo, cada uno cuenta y nos incumbe a todos actuar a favor de la coexistencia pacífica.

Dicho esto, tanto en iYa estamos! como en ¡Más allá!, los elementos culturales endógenos coexisten con sus correspondientes españoles, hispanoamericanos y guineo ecuatorianos. No se puede aprender una LE sin tener en cuenta su cultura ni la cultura de los alumnos que la aprenden, por lo que la aculturación del sujeto aprendiente y la enculturación del objeto aprendido van de la mano y se deben asumir plenamente. 
Referencias

Álvarez González, S. (2010). La relevancia del enfoque intercultural en el aula de lengua extranjera. Revista Nebrija de Lingüística Aplicada, 9, 1-15.

Barros García, B. y Kharnásova, G. M. (2012). La interculturalidad como macrocompetencia en la enseñanza de lenguas extranjeras: revisión bibliográfica y conceptual. Porta Linguarum, 18, 97-114.

Bayoko, A. S. (2021). Rasgos morfosintácticos y lexicales del español de Costa de Marfil. Revue Nzassa, Número especial, 199-208.

Bekale, H. D. et al. (2020). Ampliación de horizontes: materiales didácticos ele en África al sur del Sahara. marcoELE. Revista de Didáctica Español Lengua Extranjera, 30, 1-17.

Castellano Actual (2015). Felicidades y felicitaciones. Castellanoactual. http://udep.edu.pe/castellanoactual/felicidades-y-felicitaciones/

Chaves Salas, A. L. (2001). Implicaciones educativas de la teoría sociocultural de Vigotsky. Educación, vol. 25, núm. 2, 59-65.

Djandué, B. D. (2019). Evaluación del profesorado marfileño de enseñanza media y secundaria: percepciones y expectativas de los interesados. Práctica Docente, vol. 1, número 2, 9-23.

Djandué, B. D. (2018). De la interlengua de los usuarios marfileños de ELE a un español marfileño asumible. En W. J. Ekou y J. M. Zarandona (eds.), España en contacto con África, sus pueblos y sus culturas (pp. 127-140). Tradhuc/ Afrilenguas.

Djandué, B. D., Djè, A. M. y Kumon, A. S. (2019). De Horizontes a iYa estamos!: Lo que ha cambiado. Revue Nzassa, 2, 93-105.

Djè, A. M. (2014). Les représentations de l'Espagne et de l'Amérique hispanique dans les manuels d'espagnol en Côte d'Ivoire de 1960 à 2007. Tesis doctoral: Université François-Rabelais.

Djè, A. M., Kumon, A. S. y Djandué, B. D. (2020). África e Hispanoamérica en el manual «¡Ya estamos!» de Costa de Marfil: anatomía de una representación. En J.-A. Yao (coord.), Áfricas, Américas y Caribes Representaciones colectivas cruzadas [siglos XIX-XXI] (pp. 349-360). Servicio de Publicaciones de la Universidad de Alcalá de Henares.

Fernández Palop, M. P. y Caballero García, P. A. (2017). El libro de texto como objeto de estudio y recurso didáctico para el aprendizaje: fortalezas y debilidades. Revista Electrónica Interuniversitaria de Formación del Profesorado, 20 (1), 201-217.

García Benito, A. B. (2009). La competencia intercultural y el papel del profesor de lengua extranjera. Centro Virtual Cervantes, 493-505.

Hernández Méndez, E. y Valdez Hernández, S. (2010). El papel del profesor en el desarrollo de la competencia intercultural. Algunas propuestas didácticas. Decires, Revista del Centro de Enseñanza para Extranjeros, vol. 12, núm. 14, 91-115.

Meless, I. A. et al. (2020): ¡Más allá! Espagnol Première. Nouvelles Éditions Ivoiriennes/Centre d’Édition et de Diffusion Africaines.

Moreno Fernández, F. (2004). El contexto social y el aprendizaje de una L2/LE. En J.

Sánchez Lobato e I. Santos Gargallo (dir.), Vademécum para la formación de profesores: Enseñar español como segunda lengua (L2), LE (LE) (287-304). SGEL. 
Niango, A. C. et al. (2018). iYa estamos! Espagnol 4e. Nouvelles Éditions Ivoiriennes/Centre d'Édition et de Diffusion Africaines.

Niango, A. C. et al. (2019). iYa estamos! Espagnol 3e. Nouvelles Éditions Ivoiriennes/Centre d'Édition et de Diffusion Africaines.

Níkleva, D. G. (2009). La convivencia intercultural y su aplicación a la enseñanza de lenguas extranjeras. Ogigia, revista electrónica de estudios hispánicos, 5, 29-40.

Pérez Francés, M. J. (2002). Interculturalidad vs aculturación. Pedagogía Magna, 11, 393-397.

Rodríguez Díez, J. (2004). Transculturación, interculturación, inculturación (enculturación). Religión y cultura, L, 19-42.

Solís, C. L. (2012). La enseñanza de la competencia intercultural en el aula de E/LE:

Consideraciones didácticas para programas de inmersión lingüístico-cultural (PILC). Revista Nebrija de Lingüística Aplicada, 11, 1-12.

VV. AA. (1998). Horizontes. Espagnol 4ème. EDICEF/NEI.

VV. AA. (1999). Horizontes. Espagnol 3ème. EDICEF/NEI.

VV. AA. (2000). Horizontes. Espagnol 2de. EDICEF/NEI.

VV. AA. (2001). Horizontes. Espagnol 1ère. EDICEF/NEI.

VV. AA. (2002). Horizontes. Espagnol Terminale. EDICEF/NEI.

Yao, A. T. E. D. et al. (2020). ¡Más allá! Espagnol Seconde. Nouvelles Éditions Ivoiriennes/Centre d’Édition et de Diffusion Africaines.

Yao, A. T. E. D. et al. (2020). ¡Más allá! Espagnol Terminale. Nouvelles Éditions Ivoiriennes/Centre d'Édition et de Diffusion Africaines. 